\title{
Theoretical Description of the Glass Preparation with the Necessary Optical Properties
}

\author{
Victor Kurasov \\ Saint Petersburg State University, Universitetskaya Embankment 7/9, Saint Petersburg 199034, Russia \\ Correspondence should be addressed to Victor Kurasov; victor_kurasov@yahoo.com
}

Received 27 July 2015; Revised 27 December 2015; Accepted 29 December 2015

Academic Editor: José Luís Santos

Copyright (C) 2016 Victor Kurasov. This is an open access article distributed under the Creative Commons Attribution License, which permits unrestricted use, distribution, and reproduction in any medium, provided the original work is properly cited.

\begin{abstract}
The method to get the glass media with necessary optical characteristics is proposed. This method is based on inserting a necessary number of heterogeneous centers of given activity into the system. The theoretical description of the nucleation process in such situation is given and the resulting formulas allow choosing the characteristics of heterogeneous centers necessary to get the required optical characteristics of the media.
\end{abstract}

\section{Introduction}

The theory of a vitreous state inevitably includes the nucleation phenomena [1]. Every vistreous medium contains the embryos of a crystal phase which are growing extremely slowly. So one can say that they are practically stable objects. Such embryos are the centers of scattering and they affect the optical properties of glass. The start of systematic scattering theory was given by Lord Rayleigh [2]; the scattering on fluctuations was considered by Smoluchowsky and Einstein $[3,4]$. Essential contribution in the consideration of the scattering with account of magnetic properties was made by Cabannes $[5,6]$. A bright example of the theory of scattering on particles of essential sizes is given by the famous Mie scattering theory [7]. This approach was essentially generalized by Jobst [8] and Debye [9].

In every scattering theory the distribution of the embryos sizes is reflected in the optical coefficients of material. Ordinary it is necessary to know only several first momentums of the distribution of the embryos over sizes. Sometimes it is sufficient to know only the mean size of the embryos.

Impurities are inevitably presented in the media and they are like some heterogeneous centers. The nucleation theory on heterogeneous centers was investigated already by Frenkel in [10] and this paper completes the classical theory of nucleation created by Becker and Doering [11], Volmer [12], Kramers [13], and Zeldovich [14]. The full analysis of the stationary nucleation rate in the case of heterogeneous condensation was given by Kuni [15]. Even without the solution of kinetic equation one can evidently see that the main factor in the rate of nucleation is the Boltzmann distribution $\exp (-F)$, where $F$ is the free energy of the critical embryo counted in the thermal units. This value is the central object of the theory and the main efforts were paid to find a correct expression for $F$. Unfortunately it is rather difficult to do and even in the simplest case of the charged nuclei it is necessary to build a rather complex theory with decompositions [1618]. These decompositions were constructed in frames of the general Gibbs dividing surfaces theory [19]. In the formalism of these decompositions there appear several coefficients with very specific physical meaning.

During the process of the glass preparation it is necessary to have the glass with the necessary optical properties. So it is necessary to have impurities in the necessary quantity and of the given size.

Around the embryos there appear specific profiles of density of the substance. The role of the density profiles in the light propagation has been studied in [20]. The analogous property in frames of the embryos in nucleation theory was investigated in [21].

The classical theory of nucleation $[22,23]$ is like an elegant necklace which has to be around the free energy of critical embryo. The problem here is not only in the necessity to know what the surface tension has to be put in these 
constructions [24] and to describe the surface layer [25], but also to know the influence of the heterogeneous center on these objects. Even in the case without the heterogeneous center the question of the free energy profile is one of the most actual questions [26]. Considering the possibility of the direct measurement of the free energy of embryos one has to state that except special situations (see [27]) there is no clear way to do it. The direct application of the statistical mechanical approach [28] also causes certain difficulties. The same can be said about the comparison with the $2 \mathrm{D}$ Ising model as it is proposed in [29]. The density functional approach [3033] also can not give the absolutely precise result. But the accuracy of the last approach is rather satisfactory. One has to mention also the possibility of using the approximate scaling formulas [34-36] based on some characteristic basic points for decompositions as parameters. One can also insert here the generalizations associated with the dependence of the surface tension on the size $[37,38]$, the effects in constrained systems [39], and the deformation of heterogeneous centers [40]. Here the possibility of fluctuations [41] and the Tolmanlike corrections [42] is not considered. The refinements of $[43,44]$ can be introduced here directly.

The most evident way to get the necessary properties is to govern the law of creation of the ideal metastability $\Phi$, that is, the imaginary metastability which would be in the system when the process of the new phase formation is forbidden. The value of the ideal metastability is fully governed externally and it seems that this function can be regulated to have the necessary properties. Unfortunately, the preparation of the glass requires the absolutely prescribed conditions to have the glass with optimal properties and it is impossible to change the conditions of the glass preparation. So $\Phi$ as a function of time $t$ is supposed to be fixed.

But one can propose another rather simple and effective method. The main idea is to inject a given number of heterogeneous centers of the given activity to change the effective conditions of the nucleation of the main quantity of impurities which occurs pseudo-homogeneously (i.e., without exhaustion of the potential sites). The theoretical description of this method will be the matter of the current paper. This paper is organized as follows:

(i) At first the properties of the nucleation rate will be recalled. The exponential approximation for the nucleation rate as a function of a supersaturation will be introduced.

(ii) To construct the description of the multistage process which is the nucleation with the presence of the active heterogeneous impurities at first the nucleation without heterogeneous impurities will be studied. This process is more simple but the extraction of the characteristic features will help in construction of the more complex process.

(iii) Having started the description of the nucleation process with the presence of the active heterogeneous impurities one can consider the separate process of exhaustion of these impurities or the formation of the embryos on the active heterogeneous impurities. This part of consideration will give the parameters of the influence of the embryos on heterogeneous centers on the process of formation of the pseudohomogeneous embryos of a crystal phase.

(iv) Under the influence of heterogeneously formed embryos the formation of the pseudo-homogeneous embryos will be studied. Namely, this part of consideration will give the necessary optical characteristics of the media.

(v) To get the necessary optical characteristics it is necessary to formulate the conditions on parameters of the active heterogeneous centers. This is the matter of the next section. Here the ways to solve the system of algebraic equations of parameters are also discussed.

(vi) The final section of the paper is Conclusions where the results are summarized and the limitations and restrictions of the theory are described.

\section{Properties of the Nucleation Rate}

The rate of nucleation $J$ is proportional to exponent of the free energy of the critical embryo. This factor is the main factor and that is why the free energy $F$ of the critical embryo is the main object of interest in the investigations devoted to the nucleation.

The general formula for the rate of nucleation is the following:

$$
J=n_{1} \exp (-F) Z
$$

In (1) $n_{1}$ is the number of sites (or the molecules) which can be the starting point for the nucleation (crystallization) formation of the embryo and the factor $Z$ is the Zeldovich factor. The value of $Z$ is given by the numerous considerations but the approach appearing in the classical theory of nucleation is not yet radically reconsidered. Although there are some attempts to refine the Zeldovich factor these refinements do not lead to the change of this value in the order of the magnitude. Some interesting ideas for $Z$ appear in the multidimensional consideration $[45,46]$ but this is not the subject of the current paper.

It is reasonable to extract in $Z$ the kinetic factor $W_{c}^{+}$(the number of adsorbed molecules in the time unit) and to write

$$
Z=Z^{+} W_{c}^{+}
$$

speaking about the truncated Zeldovich factor $Z^{+}$because contrary to the liquid-vapor nucleation or the solid-liquid nucleation in the situation considered here (i.e., in the process of vitrification) the kinetic factor has specific behavior: at some moment of time it becomes so small that even the supercritical embryos practically stop growing. Otherwise it is impossible to explain why the crystal embryos do not gradually transform the whole volume of the glass into the set of crystals.

In the traditional approach mentioned above it is supposed that the intensity of absorption of new molecules by the embryo $W^{+}$is

$$
W^{+}=\frac{A}{4} n_{\text {near }} v_{t} K,
$$


where $n_{\text {near }}$ is the density of the molecules in the vicinity of the embryo, $v_{t}$ is the mean thermal velocity of the molecule, $K$ is the condensation coefficient, and $A$ is the surface area of the embryo.

The last formula (3) is supposed to be valid both for the critical and for the supercritical embryos. The evolution of undercritical embryos is not too interesting because in the region of undercritical embryos there is the quasiequilibrium state.

Really, formula (3) has a rather general range of application. Then it follows that the peculiarities of $W^{+}$are caused by the peculiarities of $K$. This explains the factorization (2). The condensation coefficient $K$ contains the main dependence specific for the vitrification process. Ordinary it is presented as

$$
K=\exp (-\Pi)
$$

implying that in the process of adsorption there is a necessity to overcome some barrier. The height of barrier is denoted by $\Pi$. This barrier can have a very specific origin including the barriers for displacement of the molecules in the environment of the embryo in order to give enough space for the appropriate installation of the new molecule in the embryo (this seems to be the main reason for annulation of $K$ ). So only some general remarks can be made concerning (4).

Instead of the previous formula (4) it is better to write

$$
K=K_{0} \exp (-\Pi)
$$

implying that even when the thermodynamic conditions correspond to the absence of imaginary barrier there remain other factors (like thermal relaxation, noncorrespondence of the position of the molecule and the profile of the adsorbing region of the embryo, etc.) which affects the probability of adsorption. Note that barrier in (5) is an abstract effective barrier.

In the liquid-vapor nucleation it is reasonable to suppose that the explicit dependence of $K$ on the time $t$ is rather smooth. In the vitrification process $K$ has to go to zero when $t$ grows. It is rather easy to explain why it is so. It is supposed that ordinary there are no thermal effects of nucleation and the creation of $\Phi$ is attained by the cooling of the system. Then the vapor consumption leads to the decrease of the number $n$ of the molecules but the temperature $T$ is not affected by embryos. The decrease of $T$ ordinary leads to increase of the activation barriers. If it is supposed that $K \sim \exp (-\Pi)$, where $\Pi$ is the activation barrier of accommodation, then we need the increase of $\Pi$ with decrease of temperature. Really, it is quite natural because the activation barrier is measured in thermal units. Then it can be seen that $K$ goes to zero.

If $\Pi$ is linearized as a function of $t$ then it is possible to come to

$$
K \sim \exp \left(-k_{\pi} t\right)
$$

with the linearization coefficient $k_{\pi}$. The linearization (6) invokes a question of the dependence of $\Phi$ on time. Here it is also supposed that the linearization can be made and one can come to

$$
\Phi \approx \phi t+\Phi_{0}
$$

with parameters $\phi$ and $\Phi_{0}$. Parameter $\Phi_{0}$ can be put to zero by the appropriate shift of the time scale.

Certainly, it is necessary to have some concrete dependence in order to present some concrete formulas. Analogous theory can be constructed for some other concrete dependence of $\Phi$ on $t$.

Certainly $K$ can not be greater than 1 . The exponential approximation does not correspond to this property. A more simple and effective approximation is the following: $K=K_{0}$ for $t<t_{0}$ and $K=0$ for $t>t_{0}$. Namely, this approximation will be used. Fortunately it is possible to determine $t_{0}$, at least to estimate $t_{0}$ in order to ensure the correct final size of embryos.

One has to stress that $K$ appears both in expression for $J$ and in expression for the rate of growth. So after $t_{0}$ there will be no appearance of the supercritical droplets nor growth of the already existing droplets. The process is therefore stopped.

Since it is required that the system is metastable it means that it is supposed that without active heterogeneous centers there will be some embryos of the new phase. This means that

$$
t_{0}>t_{+},
$$

where $t_{+}$is the moment of intensive formation of the pseudohomogeneous embryos. Certainly the moment $t_{*}$ in (8) of characteristic time of appearance of embryos on the active heterogeneous centers has to be smaller than $t_{+}$. Then

$$
t_{*}<t_{+}<t_{0}
$$

One can prove that the moment $t_{* *}$ of the pseudohomogeneous formation in the presence of active heterogeneous centers satisfies inequality

$$
t_{* *}<t_{+}
$$

Then

$$
t_{* *}<t_{0} .
$$

Inequalities (8), (9), (10), and (11) presented above are rather important because they allow stating that the process of nucleation can be considered at the practically constant value of $K$.

A special question which has to be analyzed here is whether the rate of nucleation is really the stationary one. Justifications of the stationarity are quite similar to the liquidvapor case.

The mechanism of the embryos growth can be chosen as the free molecular one which corresponds to the case of crystallization. This leads to $n_{\text {near }}=n$, where $n$ is the mean concentration of the molecules in the noncrystalline phase.

The rate of nucleation $J$ is the function of the power of metastability $\zeta$ which is called the supersaturation. Ordinary $\zeta$ is presented as

$$
\zeta=\frac{n}{n_{\infty}}-1
$$

where $n_{\infty}$ is the concentration of molecules in the noncrystalline phase at the state of the phase coexistence. Certainly then

$$
\zeta=\Phi-G
$$


where $G$ is the number of the molecules in the crystalline phase calculated in units of $n_{\infty}$. Because of the growth of the crystals the value $G$ grows also. At $t_{0}$ the value $G$ stops to grow but the process of the new droplets formation stops also.

One can see that according to (12), (13) the supersaturation $\zeta$ is not governed purely externally but depends on the process of nucleation and on the process of the substance consumption and the media heating by the regular growth of supercritical embryos. So there appears the self-consistent problem which is the subject of investigation in the theories of the global nucleation.

To see how $J$ depends on $\zeta$ it is reasonable to notice that the main dependence occurs through the factor $\exp (-F)$. The free energy $F$ is the smooth regular function of thermodynamic parameters and of $\zeta$ and it is reasonable to linearize it on the deviation in $\zeta$. It leads to

$$
J(\zeta)=J\left(\zeta_{0}\right) \exp \left(\Gamma \frac{\zeta-\zeta_{0}}{\zeta_{0}}\right)
$$

where $\zeta_{0}$ is some base for decomposition and $\Gamma$ is a parameter of linearization. For $\Gamma$ in (14) one can get

$$
\Gamma=\left.\zeta_{0} \frac{d F}{d \zeta}\right|_{\zeta=\zeta_{0}}=\left.\zeta_{0} \nu_{c} \frac{d \mu}{d \zeta}\right|_{\zeta=\zeta_{0}},
$$

where $\mu$ is the chemical potential and $\nu_{c}$ is the number of the molecules in the critical embryo. The function $\zeta_{0} d \mu / d \zeta$ is rather smooth and takes moderate values. For $\mu$ of the mother phase system like the ideal gas (i.e., for every mother phase where the mean field approximation works) one can easily come to

$$
\left.\zeta_{0} \frac{d \mu}{d \zeta}\right|_{\zeta=\zeta_{0}}=\frac{\zeta_{0}}{\zeta_{0}+1}
$$

So according to (15), (16) one can estimate $\Gamma$ as $\nu_{c}$ and it is possible to see that $\Gamma$ gets the big values $\left(\nu_{c} \gg 1\right.$ to ensure the thermodynamic description of the critical embryo). It means that the dependence of $J$ on $\zeta$ is rather sharp.

Later the mentioned estimate leads to the estimate

$$
\frac{\left|\zeta-\zeta_{!}\right|}{\zeta_{!}} \sim \nu_{c}^{-1}
$$

during the period of intensive nucleation. Here $\zeta_{!}$is the value of $\zeta$ at some characteristic moment which belongs to the nucleation period.

Also one can get the estimate analogous to (17) for $\Phi$

$$
\frac{\left|\Phi-\Phi_{!}\right|}{\Phi_{!}} \sim \nu_{c}^{-1}
$$

and on the base of (18) one can see that also

$$
\frac{\left|t-t_{!}\right|}{t_{!}} \sim v_{c}^{-1} .
$$

The last estimate shows that the nucleation period is relatively short in time. Namely, estimate (19) allows speaking above about $t_{+}, t_{*}$, and $t_{* *}$ as some moments of time for nucleation.

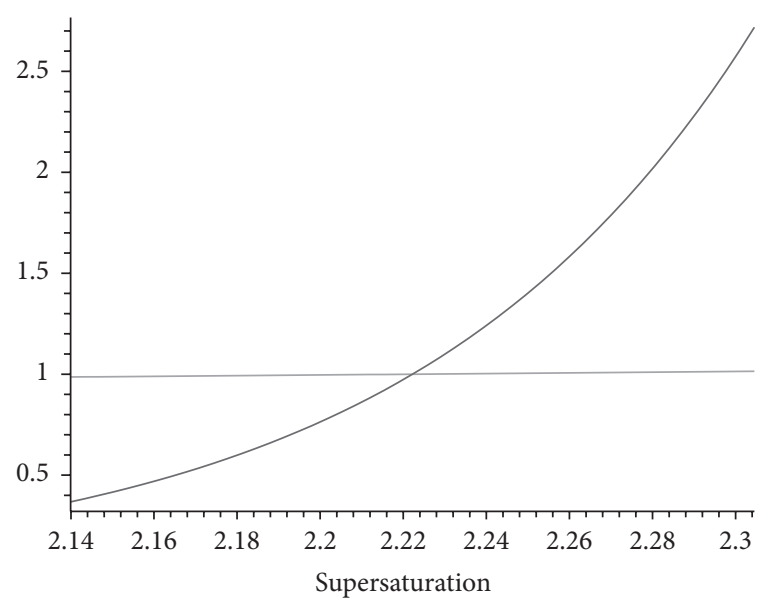

FIGURE 1: Behavior of the exponential and preexponential factors for the nucleation rate.

To see that the exponential approximation really works one can present the characteristic behavior of the Zeldovich factor $Z^{+}$for the pseudo-homogeneous case. It is shown in Figure 1. One can see here two lines: the practically constant line which is the reduced Zeldovich factor and the exponent which is the main Boltzmann factor. So one can see that the preexponential factor (Zeldovich factor) does not really change. Here the worst value for the parameter $\Gamma$ is $\Gamma=27$ and the worst value for the renormalized surface tension $\sigma$ is $\sigma=10$ (the biggest possible value in the normal conditions). Namely, these values are taken for this example. Certainly, one can not include here the explicit time dependence of the kinetic coefficient of adsorption.

Really, the relative change of the preexponential factor here has the order $1 / 27=\Gamma^{-1}$. It can be hardly seen in Figure 1 because the variation is very small.

\section{Nucleation without Heterogeneous Impurities}

To calculate $G$ one has to know not only the rate of nucleation $J$ but also the rate of the embryos growth. Fortunately the free molecular regime leads to the following rather simple rate of growth of the number $v$ of the molecules inside the supercritical embryo:

$$
\frac{d \nu^{1 / 3}}{d t}=\frac{\zeta}{\tau}
$$

with characteristic parameter $\tau$. Certainly,

$$
\tau \sim K_{0}^{-1} .
$$

Namely, (21) shows that the time $t_{0}$ of freezing stops the growth.

One can prove that in calculation of $G$ only the input from the supercritical embryos has to be taken into account.

Fortunately $d v^{1 / 3} / d t$ according to (20) is rather unsensitive to the supersaturation and there is no need to observe 
the influence of the nucleation on the rate of the embryos growth. Then for the embryo appeared at $t^{\prime}$ the number of molecules will be approximately

$$
v=\left(\frac{\zeta}{\tau}\right)^{3}\left(t-t^{\prime}\right)^{3}
$$

and with account of (17) one can transform (22) into

$$
v=\left(\frac{\zeta_{!}}{\tau}\right)^{3}\left(t-t^{\prime}\right)^{3}
$$

On the base of (23) the expression for $G$ will be

$$
G(t)=\int_{-\infty}^{t} d t^{\prime}\left(t-t^{\prime}\right)^{3} \frac{J\left(t^{\prime}\right)}{n_{\infty}}\left(\frac{\zeta_{!}}{\tau}\right)^{3}
$$

Then balance equation (13) on the base of (24) can be written as

$$
\zeta=\Phi-\int_{-\infty}^{t} d t^{\prime}\left(t-t^{\prime}\right)^{3} \frac{J\left(t^{\prime}\right)}{n_{\infty}}\left(\frac{\zeta_{!}}{\tau}\right)^{3}
$$

Having inserted the mentioned linearizations and exponential approximation (14), (15) for $J$ it is possible to come in (25) to

$$
\begin{aligned}
& \zeta(t)=\phi t-\frac{J\left(\zeta\left(t_{+}\right)\right)}{n_{\infty}} \\
& \quad \cdot \int_{-\infty}^{t} d t^{\prime}\left(t-t^{\prime}\right)^{3} \exp \left(\Gamma \frac{\left(\zeta\left(t^{\prime}\right)-\zeta_{+}\right)}{\zeta_{+}}\right)\left(\frac{\zeta_{+}}{\tau}\right)^{3} .
\end{aligned}
$$

Expression (26) has enough high accuracy.

One can prove that

$$
\frac{\left|\Phi_{+}-\zeta_{+}\right|}{\Phi_{+}} \sim v_{c}^{-1}
$$

Estimate (27) allows taking $\Phi_{+}$instead of $\zeta_{+}$and writing (26) as

$$
\begin{aligned}
& \zeta(t)=\phi t-\frac{J\left(\Phi\left(t_{+}\right)\right)}{n_{\infty}} \\
& \cdot \int_{-\infty}^{t} d t^{\prime}\left(t-t^{\prime}\right)^{3} \exp \left(\Gamma \frac{\left(\zeta\left(t^{\prime}\right)-\Phi_{+}\right)}{\Phi_{+}}\right)\left(\frac{\Phi_{+}}{\tau}\right)^{3} .
\end{aligned}
$$

Having shifted the time to have the zero point at $t_{+}$one can come instead of (28) to

$$
\begin{aligned}
& \zeta(t)=\phi t+\Phi_{+}-\frac{J\left(\Phi\left(t_{+}\right)\right)}{n_{\infty}} \\
& \cdot \int_{-\infty}^{t} d t^{\prime}\left(t-t^{\prime}\right)^{3} \exp \left(\Gamma \frac{\left(\zeta\left(t^{\prime}\right)-\Phi_{+}\right)}{\Phi_{+}}\right)\left(\frac{\Phi_{+}}{\tau}\right)^{3} .
\end{aligned}
$$

The last equation (29) is typical for nucleation and is formulated, for example, in [47] as one of the limit cases. It can be solved by iterations

$$
\begin{gathered}
\zeta_{(i+1)}(t)=\phi t+\Phi_{+}-\frac{J\left(\Phi\left(t_{+}\right)\right)}{n_{\infty}} \int_{-\infty}^{t} d t^{\prime}\left(t-t^{\prime}\right)^{3} \\
\cdot \exp \left(\Gamma \frac{\left(\zeta_{(i)}\left(t^{\prime}\right)-\Phi_{+}\right)}{\Phi_{+}}\right)\left(\frac{\Phi_{+}}{\tau}\right)^{3} .
\end{gathered}
$$

The lower index in brackets indicates the number of iteration. The initial approximation in the iteration procedure (30) is

$$
\zeta_{(0)}=\Phi_{+}+\phi t .
$$

The first approximation calculated on the base of (31) is

$$
\begin{aligned}
& \zeta_{(1)}(t)=\phi t+\Phi_{+}-\frac{J\left(\Phi\left(t_{+}\right)\right)}{n_{\infty}}\left(\frac{\Phi_{+}}{\tau}\right)^{3} \\
& \cdot \int_{-\infty}^{t} d t^{\prime}\left(t-t^{\prime}\right)^{3} \exp \left(\Gamma \frac{\phi t^{\prime}}{\Phi_{+}}\right) .
\end{aligned}
$$

Since

$$
\begin{gathered}
\int_{-\infty}^{t} d t^{\prime}\left(t-t^{\prime}\right)^{3} \exp \left(\Gamma \frac{\phi t^{\prime}}{\Phi_{+}}\right) \\
=\exp \left(\Gamma \frac{\phi t}{\Phi_{+}}\right)\left(\Gamma \frac{\phi}{\Phi_{+}}\right)^{-4} 6
\end{gathered}
$$

it is easy to come in (32) on the base of (33) to

$$
\begin{aligned}
\zeta_{(1)}(t) & \\
= & \phi t+\Phi_{+} \\
& -\frac{J\left(\Phi\left(t_{+}\right)\right)}{n_{\infty}}\left(\frac{\Phi_{+}}{\tau}\right)^{3} \exp \left(\Gamma \frac{\phi t}{\Phi_{+}}\right)\left(\Gamma \frac{\phi}{\Phi_{+}}\right)^{-4} 6 .
\end{aligned}
$$

Equation (34) has enough high accuracy.

One can prove that already the first approximation is rather good and the size spectrum

$$
J(t)=J\left(\Phi_{+}\right) \exp \left(\Gamma \frac{\left(\phi t-\left(J\left(\Phi\left(t_{+}\right)\right) / n_{\infty}\right)\left(\Phi_{+} / \tau\right)^{3} \exp \left(\Gamma\left(\phi t / \Phi_{+}\right)\right)\left(\Gamma\left(\phi / \Phi_{+}\right)\right)^{-4} 6\right)}{\Phi_{+}}\right)
$$

is rather accurate. 


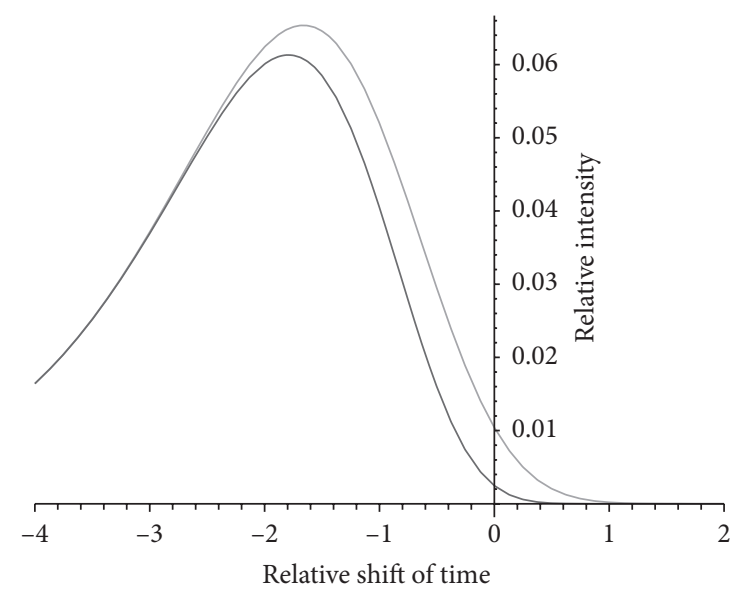

FIgURE 2: The fist and the second iterations for the nucleation rate as a function of the time shift. Here the nucleation rate is drawn in special renormalized units.

The rate of nucleation in the first (see (35)) and the second approximations of the iteration procedure is drawn in Figure 2. One can see here two lines: the lower line is the first iteration and the upper line is the second iteration for the nucleation rate as a function of the special shifted and scaled time.

One can see that the first iteration is rather close to the second one. One can see the similarity of the forms of the first and the second iteration. If it is necessary to increase the accuracy one can make a simple shift.

One can choose $t_{+}$as the moment of the maximal supersaturation. Then

$$
\phi=\frac{J\left(\Phi\left(t_{+}\right)\right)}{n_{\infty}}\left(\frac{\Phi_{+}}{\tau}\right)^{3}\left(\Gamma \frac{\phi}{\Phi_{+}}\right)^{-3} 6
$$

is the algebraic equation on parameters (actually on $\Phi_{+}$) and the good approximation to solve (36) is

$$
\phi=\frac{J\left(\Phi\left(t_{+}\right)\right)}{n_{\infty}} \tau^{-3}\left(\frac{\Gamma}{\phi}\right)^{-3} 6 .
$$

Approximation (37) is rather accurate.

All what has been done is necessary to show that $G$ at $t_{+}$, that is, $G_{+}$, can be estimated as

$$
G_{+}=\Gamma_{+}^{-1} \Phi_{+}=\nu_{c}\left(t_{+}\right)^{-1} \Phi_{+} .
$$

Although (38) is rather simple it will be absolutely sufficient for further constructions.

Now the further evolution will be considered. One can see there two periods. The first period is the rapid consumption of the surplus substance

$$
G_{\text {surplus }}=\Phi_{+}\left(1-\Gamma_{+}^{-1}\right) .
$$

It is finished at some moment of time $t_{1+}$ when the whole (or practically all) surplus substance $G_{\text {surplus }}$ is in the embryos. Now one has to estimate $t_{\mid+}$. It is possible to see on the base of (39) that

$$
t_{1+}-t_{+} \sim \Gamma^{-2 / 3} t_{+}
$$

So according to (40) this period is rather rapid. It allows supposing that the condition $t_{0}>t_{+}$also ensures

$$
t_{0}>t_{1+}
$$

The probability that (41) is not observed is lower than $\Gamma^{-2 / 3} \ll$ 1.

Later the supersaturation is fallen and there will be an asymptotic period where $G \approx \Phi$. Here the mean effective linear coordinate $L$ of the spectrum grows very slow like $L \sim t^{1 / 3}$. At $t_{0}$ it stops to grow. It is reasonable to put

$$
L\left(t_{0}\right) \approx L\left(t_{\mid+}\right)
$$

and (42) gives the natural condition for the mean linear size of the embryos.

Here one has to make one important notation. In the liquid-vapor transition the volume $v_{v}$ per molecule in a vapor phase is a thousand times greater than the volume per particle $v_{l}$ in a liquid phase. This ensures that the relative volume of the new phase in vapor-liquid transition is small.

In the opposite liquid-vapor transition it seems that the situation will be opposite and the whole volume begins to be the vapor phase. But here the main effect of the bubble formation is to take away the surplus stretching. It is necessary to keep in mind.

In the situation of the liquid-crystal transition the volume $v_{k}$ per the molecule in a crystal phase is comparable with $v_{l}$ and it is possible to see the crystallization of the whole volume (ordinary this does not take place because of the heat release effects). But in the process of vitrification the mother phase is so viscous that the main effect of the appearance of the crystal embryo is to take away the stretching and the tension in the mother media. Then the relative volume of the new crystal phase will be small at $t_{\mid+}$.

Later without $t_{0}$ the crystal phase would cover practically the whole volume. But the cut-off of the nucleation and condensation process at $t_{0}$ will stop the consumption of the volume by the crystal phase. Unfortunately one can not radically change $\Phi(t)$ and $t_{0}$. That is why the method of heterogeneous centers insertion is suggested.

\section{Formation of the Embryos on the Active Heterogeneous Impurities}

Now the process of the heterogeneous condensation will be studied. It is supposed that the centers are relatively active. This means that all of them are exhausted in the process of nucleation. This property simplifies the theoretical description of kinetics of the phase formation.

One can note that the attribution of the centers (sites) to the group of the active ones depends on the intensity of the change of the external conditions. So this property is not absolute.

It is supposed also that the embryos on the heterogeneous centers can be described thermodynamically. It means that the number of molecules $\nu_{c}$ in the critical embryo is big enough. As for the number $v_{e}$ of the molecules inside the equilibrium center one can avoid the restriction $\nu_{e} \gg 1 \mathrm{nec}-$ essary for the thermodynamic description of the equilibrium 


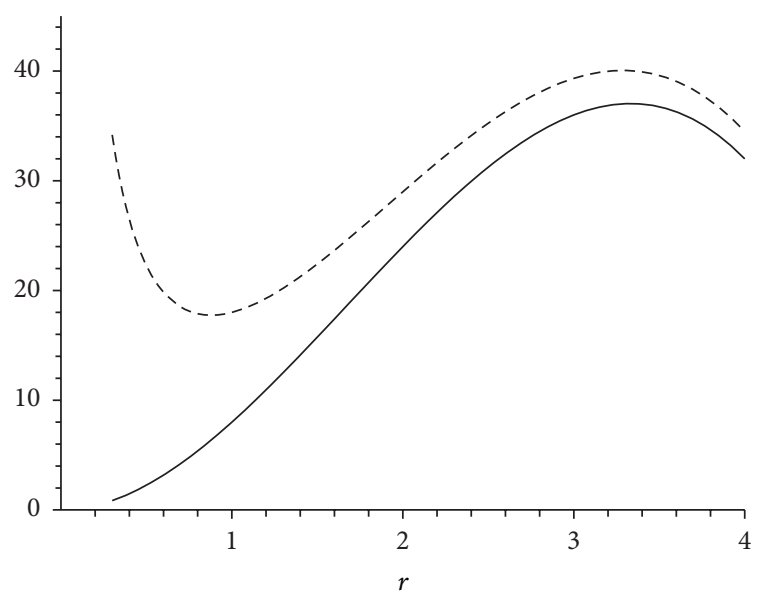

FIGURE 3: Heterogeneous and homogeneous free energies.

embryo. It can be done since only the behavior of the height of the activation barrier

$$
\Delta F=F\left(\nu_{c}\right)-F\left(\nu_{e}\right)
$$

is interesting and it determines the results of nucleation. Here the variation of $F\left(v_{e}\right)$ because of uncertainty of deviation of the embryos description from the thermodynamic one will be small in comparison with $\Delta F$.

The behavior of the heterogeneous embryo free energy together with the behavior of the homogeneous free energy is drawn in Figure 3. The solid line is the homogeneous free energy and the dashed line is the heterogeneous free energy. Here the capillary model

$$
F=\sigma r^{2}-\ln (\zeta+1) r^{3}
$$

for $r=v^{1 / 3}$ is used for the homogeneous case. As in Figure 1 the values $\sigma=10$ and $\zeta \approx 2$ are used. In the heterogeneous case

$$
F=\sigma r^{2}-\ln (\zeta+1) r^{3}+\frac{\widetilde{c}}{r} .
$$

Here $\widetilde{c}=10$ is used as a value comparable with $\sigma$. Certainly, this example (namely, (44), and (45)) is no more than a model.

Since one can prove that

$$
\begin{aligned}
& \frac{d F_{c}}{d \mu}=v_{c}, \\
& \frac{d F_{e}}{d \mu}=v_{e},
\end{aligned}
$$

where $\mu$ is a chemical potential, one can see that according to (43), (46) only when $\nu_{e} \gg 1$ is the value $d F_{e} / d \mu$ essential in $d \Delta F / d \mu$. So when $\nu_{e}$ is small one can not describe the equilibrium embryo adequately but the dependence of $F_{e}$ on $\zeta$ (which means the dependence on $\mu$ ) is not important. by

For heterogeneous nucleation the nucleation rate is given

$$
\widehat{J}=n_{\text {het }} W_{c}^{+} \exp (-\Delta F) \frac{Z^{+}}{\Delta_{e} \nu}
$$

where $n_{\text {het }}$ is the number of the free heterogeneous centers (unoccupied by the supercritical embryos) and $\Delta_{e} \nu$ comes from the equilibrium distribution

$$
n^{e}=n_{\text {het }} \frac{\exp \left(-\left(F-F_{e}\right)\right)}{\Delta_{e} \nu} .
$$

So the structure of the rate of nucleation (47), (48) remains the same; it is the equilibrium distribution formally prolonged until the critical embryo and multiplied by the kinetic factor and by the truncated Zeldovich factor $Z^{+}$.

Instead of the general theory (see [47]) one can simply note that all centers will be exhausted when the ideal number of the formed droplets $N_{\text {id het }}$ which is calculated as

$$
N_{\text {id het }}=\int_{-\infty}^{t} \widehat{J}\left(\Phi\left(t^{\prime}\right), n_{\text {het }}=n_{\text {tot }}\right) d t^{\prime},
$$

where $n_{\text {tot }}$ is the total number of heterogeneous centers, is equal to $n_{\text {tot }}$. The requirement of essential exhaustion of heterogeneous centers together with (49) gives an equation

$$
\int_{-\infty}^{t} \widehat{J}\left(\Phi\left(t^{\prime}\right), n_{\text {het }}=n_{\text {tot }}\right) d t^{\prime}=n_{\text {tot }}
$$

on $t$.

To solve (50) more effectively it is reasonable to introduce an approximation

$$
\begin{aligned}
\widehat{J}\left(\Phi(t), n_{\text {het }}=n_{\text {tot }}\right) \\
\quad=\widehat{J}\left(\Phi\left(t_{*}\right), n_{\text {tot }}\right) \exp \left(\Gamma_{\text {het }} \frac{\Phi(t)-\Phi_{*}}{\Phi_{*}}\right),
\end{aligned}
$$

where

$$
\Gamma_{\text {het }}=\left.\Phi_{*}\left(\frac{d F_{c}}{d \zeta}-\frac{d F_{e}}{d \zeta}\right)\right|_{\zeta=\Phi_{*}} .
$$

The accuracy of (51), (52) is rather high.

One can easily estimate $\Gamma_{\text {het }}$ as

$$
\Gamma_{\text {het }}=\left.\Phi_{*}\left(\nu_{c}-v_{e}\right) \frac{d \mu}{d \zeta}\right|_{\zeta=\zeta_{0}} .
$$

Then (50) can be rewritten with the help of (53) as

$$
\widehat{J}\left(\Phi\left(t_{*}\right), n_{\mathrm{tot}}\right) \int_{-\infty}^{t} \exp \left(\phi t^{\prime}\right) d t^{\prime}=n_{\mathrm{tot}}
$$

and gives

$$
\widehat{J}\left(\Phi\left(t_{*}\right), n_{\text {tot }}\right)=n_{\text {tot }} \phi
$$

or

$$
W_{c}^{+} \exp \left(-\Delta F\left(\Phi_{*}\right)\right) \frac{Z^{+}}{\Delta_{e} \nu}=\phi
$$

Equation (56) does not contain $n_{\text {tot }}$. Certainly (54) and (54) do not contain it also. So the power of exhaustion and the level of exhaustion are purely determined by the nature of centers 
and do not depend on their quantity. This allows speaking about the active centers. It is possible to see that having changed the number of centers one can change the numbers of the embryos which begin to consume metastability rather actively after $t_{*}$.

The detailed justification of the presented method is rather long but it exists at the level of precise analytical derivation.

\section{Formation of \\ the Pseudo-Homogeneous Embryos}

Now the formation of the pseudo-homogeneous embryos will be studied. The difference from the consideration of the homogeneous nucleation presented above will be the change of conditions for the new process because there are the heterogeneous consumers of metastability.

Certainly, the new forming embryos consume metastability and this leads to the perturbation of the rate of growth of the supercritical embryos formed on heterogeneous centers. But one can prove that this influence is very small and can be neglected. So one can see two separate problems here.
The first problem is to determine the supersaturation which appears after the heterogeneous embryos have been formed. The second problem is to determine the characteristics of the embryos size spectrum when the homogeneous process takes place.

The balance equation with account of only heterogeneous centers is written as

$$
\phi t+\Phi_{+}=\zeta+n_{\mathrm{tot}} \frac{z(t)^{3}}{n_{\infty}}
$$

where $z(t)$ is the coordinate of the linear size of embryos and it can be found as

$$
z(t)=\tau^{-1} \int_{t_{*}}^{t} d t^{\prime} \zeta\left(t^{\prime}\right)
$$

So (57), (58) lead to the first-order differential equation which can be solved. One can reduce (57) with the help of (58) to

$$
\frac{d z}{d t}+c z^{3} t-a t=0
$$

with parameters $a$ and $c$ and get the solution of (59):

$$
t=\sqrt{\left|\frac{2}{3} \frac{\ln \left|\left(z-(a / c)^{1 / 3}\right)\right|}{c(a / c)^{2 / 3}}-\frac{1}{3} \frac{\ln \left|\left(z^{2}+z(a / c)^{1 / 3}+(a / c)^{2 / 3}\right)\right|}{c(a / c)^{2 / 3}}-\frac{2}{3} \frac{\sqrt{3} \arctan \left(\left((2 / 3)\left(z /(a / c)^{1 / 3}\right)+1 / 3\right) \sqrt{3}\right)}{c(a / c)^{2 / 3}}+C_{1}\right|}
$$

with the arbitrary constant $C_{1}$ which has to be chosen to satisfy the initial condition

$$
\left.z\right|_{t=t_{+}}=0
$$

So then (61) leads to the concrete value of the constant in (60) which will be the following:

$$
C_{1}=\sqrt{\left|\frac{2}{3} \frac{\ln \left|(a / c)^{1 / 3}\right|}{c(a / c)^{2 / 3}}-\frac{1}{3} \frac{\ln \left|(a / c)^{2 / 3}\right|}{c(a / c)^{2 / 3}}-\frac{2}{3} \frac{\sqrt{3} \arctan (((2 / 3)(1 / 3)) \sqrt{3})}{c(a / c)^{2 / 3}}\right|}
$$

Expression (62) has to be inserted into (60).

Unfortunately it is impossible to inverse the dependence $t(z)$ given by (60) and get the explicit solution. The dependence $t(z)$ for $a=1, c=1$ is drawn as an example in Figure 4.

One has to note that here it is impossible to use an approximation

$$
z(t)=\frac{\Phi_{+}\left(t-t^{\prime}\right)}{\tau}
$$

analogous to (7) but it is necessary instead of (63) to integrate $d z / d t$ explicitly.

Now the behavior of $\zeta$ will be analyzed qualitatively. The difference $\Phi-\zeta$ at first increases in time $t$ very rapidly (faster than $t^{4}$ ) and this leads to the maximum of $\zeta$ even without homogeneous appearance of embryos. After attaining the maximum of the supersaturation it decreases and the conditions for the intensive homogeneous formation of new embryos disappear. So there exist two possibilities for the further homogeneous nucleation.

The first possibility is to see the nucleation in conditions of the growing $\Psi$ which is

$$
\Psi=\Phi-n_{\mathrm{tot}} z^{3}(t) t
$$

Here in (64) the homogeneously formed embryos take part in the metastability consumption rather actively.

Alternative possibility is the following: the supersaturation $\zeta$ fully coincides with $\Psi$ and homogeneously formed embryos do not play any essential role in evolution. Here the number of embryos is

$$
N_{\text {hom }}=\int_{-\infty}^{\infty} d t^{\prime} J\left(\Psi\left(t^{\prime}\right)\right)
$$




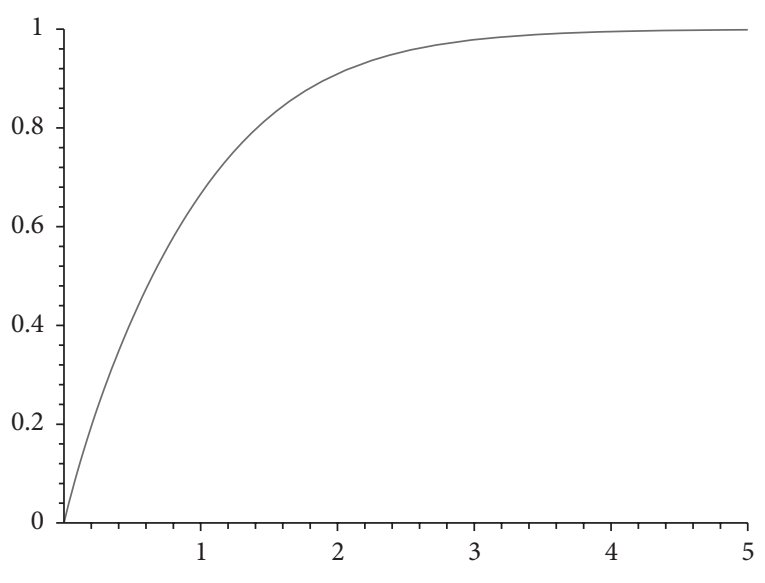

FIgURE 4: Solution $z(t)$ for $a=1, c=1$.

and the embryos linear size spectrum is

$$
f\left(\rho\left(t^{\prime \prime}\right)\right)=\frac{J\left(t^{\prime \prime}\right)}{\left(\zeta\left(t^{\prime \prime}\right) / \tau\right)}
$$

where

$$
\rho\left(t^{\prime \prime}\right)=\int_{t^{\prime \prime}}^{t} d t^{\prime} \frac{\Psi\left(t^{\prime}\right)}{\tau}
$$

for $t<t_{0}$ and

$$
\rho\left(t^{\prime \prime}\right)=\int_{t^{\prime \prime}}^{t_{0}} d t^{\prime} \frac{\Psi\left(t^{\prime}\right)}{\tau}
$$

for $t>t_{0}$. The accuracy of expressions (65), (66), (67), and (68) is sufficient.

One can use the exponential approximation based on $\max \Psi$ to calculate the number of droplets and the size spectrum. Also one can use the power decomposition of $\Psi$ at the point of maximum.

The second possibility is not interesting also from the practical point of view because it requires essential number of heterogeneous centers (sites).

The only necessary fact which remains to be clarified is the existence of the time of the end of the rapid growth $t_{1}$ of the embryos sizes. Really, the rapid increase of $z^{3} t$ can be stopped only when $\Phi$ comes to zero. But it has to be stopped because $z^{3} t$ can not be greater than $\Phi$. So $t_{1}$ exists.

How can one calculate $t_{\mid}$and the linear size at the end of intensive growth $L_{\mid}$(here these values are marked as $t_{\mid *}$ and $\left.L_{\mid *}\right)$ ? There is no sharp sensitivity of these values to the supersaturation and one can get these values by the simple iteration procedure

$$
\begin{aligned}
z(t) & =\frac{\phi}{2 \tau}\left(t^{2}-t_{+}^{2}\right), \\
\Psi & =\phi t-\left(\frac{n_{\mathrm{tot}}}{n_{\infty}\left(t_{*}\right)}\right)\left(\frac{\phi}{2 \tau}\left(t^{2}-t_{+}^{2}\right)\right)^{3} t .
\end{aligned}
$$

The time $t_{\left.\right|_{*}}$ is determined as to satisfy the following equation:

$$
\phi=\left(\frac{n_{\mathrm{tot}}}{n_{\infty}\left(t_{*}\right)}\right)\left(\frac{\phi}{2 \tau}\left(t_{1}^{2}-t_{+}^{2}\right)\right)^{3} .
$$

The value $L_{\mid *}$ is

$$
\begin{aligned}
& L_{1} \\
& \qquad \int_{t_{*}}^{t_{1}} d t\left(\phi t-\left(\frac{n_{\mathrm{tot}}}{n_{\infty}\left(t_{*}\right)}\right)\left(\frac{\phi}{2 \tau}\left(t^{2}-t_{+}^{2}\right)\right)^{3} t\right) \tau^{-1} .
\end{aligned}
$$

Expressions (70) and (71) have the necessary accuracy.

It is also necessary to determine the mean size $\widetilde{L}$ for the homogeneously formed embryos. It is

$$
\begin{aligned}
& \widetilde{L}_{\mid *} \\
& \quad=\int_{t_{m}}^{t_{1}} d t\left(\phi t-\left(\frac{n_{\text {tot }}}{n_{\infty}\left(t_{*}\right)}\right)\left(\frac{\phi}{2 \tau}\left(t^{2}-t_{+}^{2}\right)\right)^{3} t\right) \tau^{-1},
\end{aligned}
$$

where $t_{m}$ is the point of the maximum of $\Psi$. The last integral can be easily taken analytically since the integrand is the power function. Then (72) becomes a simple algebraic formula.

\section{Conditions on Parameters of the Active Heterogeneous Centers}

Now the first possibility will be considered. Here consideration is similar to the case of the pure homogeneous nucleation but it is necessary to consider $\Psi$ instead of $\Phi$.

The function $\Psi$ is determined and it is possible to approximately linearize it at the point $t_{* *}$ and to get

$$
\Psi(t)=\Psi_{* *}+\psi\left(t-t_{* *}\right),
$$

where

$$
\psi=\left.\frac{d \Psi}{d t}\right|_{t=t_{* *}} .
$$

Formula (73) (with account of (74)) is quite analogous to (7).

The balance equation is

$$
\begin{aligned}
& \zeta(t)=\psi t+\Psi_{* *}-\frac{J\left(\Psi\left(t_{* *}\right)\right)}{n_{\infty}} \\
& \cdot \int_{-\infty}^{t}\left(t-t^{\prime}\right)^{3} \exp \left(\Gamma \frac{\left(\zeta\left(t^{\prime}\right)-\Psi_{* *}\right)}{\Psi_{* *}}\right)\left(\frac{\Psi_{* *}}{\tau}\right)^{3}
\end{aligned}
$$


The last equation (75) can be solved by iterations

$$
\begin{gathered}
\zeta_{(i+1)}(t)=\psi t+\Psi_{* *}-\frac{J\left(\Psi\left(t_{* *}\right)\right)}{n_{\infty}} \\
\cdot \int_{-\infty}^{t}\left(t-t^{\prime}\right)^{3} \exp \left(\Gamma \frac{\left(\zeta_{(i)}\left(t^{\prime}\right)-\Psi_{* *}\right)}{\Psi_{* *}}\right)\left(\frac{\Psi_{* *}}{\tau}\right)^{3} .
\end{gathered}
$$

The lower index in brackets which appeared in (76) indicates the number of iteration. The initial approximation will be

$$
\begin{gathered}
\zeta_{(0)}=\Psi_{* *}+\psi t . \\
J(t)=J\left(\Psi_{* *}\right) \exp \left(\Gamma \frac{\left(\psi t-J\left(\Psi\left(t_{* *}\right)\right)\left(\Psi_{* *} / \tau\right)^{3} \exp \left(\Gamma\left(\psi t / \Psi_{* *}\right)\right)\left(\Gamma\left(\psi / \Psi_{* *}\right)\right)^{-4}\left(6 / n_{\infty}\right)\right)}{\Psi_{* *}}\right) .
\end{gathered}
$$

The first approximation on the base of (77) is

$$
\begin{aligned}
& \zeta_{(1)}(t) \\
& =\psi t+\Psi_{* *} \\
& \quad-\frac{J\left(\Psi\left(t_{* *}\right)\right)}{n_{\infty}}\left(\frac{\Psi_{* *}}{\tau}\right)^{3} \exp \left(\Gamma \frac{\psi t}{\Psi_{* *}}\right)\left(\Gamma \frac{\psi}{\Psi_{* *}}\right)^{-4} 6 .
\end{aligned}
$$

One can prove that already the first approximation (78) is
The form of the size spectrum given by (79) is rather accurate.

One can choose $t_{* *}$ as the moment of the maximal supersaturation. Then

$$
\psi=\frac{J\left(\Psi\left(t_{* *}\right)\right)}{n_{\infty}}\left(\frac{\Psi_{* *}}{\tau}\right)^{3}\left(\Gamma \frac{\psi}{\Psi_{* *}}\right)^{-3} 6
$$

is the algebraic equation on $\Psi_{* *}$. The solution of (80) is approximately

$$
\psi=\frac{J\left(\Psi\left(t_{* *}\right)\right)}{n_{\infty}} \tau^{-3}\left(\frac{\Gamma}{\psi}\right)^{-3} 6 .
$$

Formula (81) solves the problem to find parameters of the spectrum.

Now the further evolution will be considered. Since $\Psi$ already ensured $t_{1}$, here one can also see $t_{\mid}$which will be marked as $t_{\mid * *}$. One can show that

$$
\frac{\left|t_{\mid * *}-t_{* *}\right|}{t_{* *}}<\frac{\left|t_{*}-t_{\mid *}\right|}{t_{*}}
$$

if it is supposed that $\Gamma_{*}$ is equal to $\Gamma_{* *}$. So here also

$$
\frac{\left|t_{\mid * *}-t_{* *}\right|}{t_{* *}}<\Gamma_{* *}^{-1}
$$

and one can see on the base of (82), (83) that $t_{0}>t_{+}$ practically ensures $t_{0}>t_{1 * *}$.

The final values will be

$$
\begin{aligned}
& L\left(t_{0}\right) \approx L\left(t_{\mid * *}\right), \\
& \widetilde{L}\left(t_{0}\right) \approx \widetilde{L}\left(t_{\mid * *}\right) .
\end{aligned}
$$

Equations (84) demonstrate the necessary accuracy.
The value $L\left(t_{\mid * *}\right)$ is calculated as

$$
L\left(t_{\mid * *}\right) \approx L\left(t_{m}\right)
$$

where

$$
L\left(t_{m}\right)=\int_{t_{*}}^{t_{m}} \frac{\Psi\left(t^{\prime}\right)}{\tau} d t^{\prime}
$$

Approximation of (85), (86) has enough high accuracy.

The value $\widetilde{L}\left(t_{\mid * *}\right)$ is calculated as

$$
N_{\text {tothom }}\left(\widetilde{L}\left(t_{\mid * *}\right)\right)^{3}=\Psi\left(t_{* *}\right)
$$

where $N_{\text {tot hom }}$ is the total number of embryos formed homogeneously in units of $n_{\infty}\left(t_{* *}\right)$. The value $N_{\text {tot hom }}$ appearing in (87) is already determined. If one takes an approximation

$$
\widetilde{L}\left(t_{\mid * *}\right)=\left(\frac{\Psi_{* *}}{\tau}\right)\left(t_{\mid * *}-t_{* *}\right)
$$

one can get the following expression for $t_{\mid * *}$ :

$$
t_{\mid * *}=t_{* *}+\frac{\left(\Psi\left(t_{* *}\right) / N_{\text {tothom }}\right)^{1 / 3}}{\left(\Psi_{* *} / \tau\right)} .
$$

Expressions (88), (89) complete the recipe of calculations.

\section{Conclusions}

The known value of $\Phi_{* *}$ allows getting the total number of the homogeneously formed embryos as

$$
N_{\text {tothom }}=\frac{J\left(\Psi_{* *}\right)}{n_{\infty}\left(t_{* *}\right)} \int_{-\infty}^{\infty} d t^{\prime} \exp \left(\Gamma_{* *} \frac{\left(\psi t^{\prime}-J\left(\Psi\left(t_{* *}\right)\right)\left(\Psi_{* *} / \tau\right)^{3} \exp \left(\Gamma\left(\psi t^{\prime} / \Psi_{* *}\right)\right)\left(\Gamma \psi / \Psi_{* *}\right)^{-4}\left(6 / n_{\infty}\right)\right)}{\Psi_{* *}}\right) .
$$


The integration can be performed analytically which brings (90) to

$$
\begin{aligned}
& N_{\text {tothom }}=\frac{J\left(\Psi_{* *}\right)}{n_{\infty}\left(t_{* *}\right)} \frac{\Psi_{* *}}{\Gamma_{* *} \psi}\left(\Gamma_{* *} J\left(\Psi\left(t_{* *}\right)\right)\left(\frac{\Psi_{* *}}{\tau}\right)^{3}\right. \\
& \left.\cdot\left(\Gamma \frac{\psi}{\Psi_{* *}}\right)^{-4} \frac{6}{n_{\infty} \Psi_{* *}}\right)^{-1} .
\end{aligned}
$$

Simple formula (91) allows calculating $N_{\text {tothom }}$.

Changing $\triangle F$ one can choose $t_{*}$. Changing $n_{\text {tot }}$ one can choose the force of the action of heterogeneous centers and then choose $t_{* *}$. Then one can get necessary $\widetilde{L}\left(t_{0}\right)$ and $N_{\text {tot hom }}$.

The second possibility when the supersaturation is determined by the heterogeneously formed embryos is not too attractive because here the number of heterogeneous centers is greater than in the first variant and can be even greater than the number of homogeneously formed embryos. The only positive property is that in the majority of situations

$$
t_{1 *}<t_{1+}
$$

and one can hope that

$$
t_{\mid *}<t_{0} .
$$

So the process of nucleation is frozen only after the end of essential stages of nucleation. The probability of violation of (92), (93) is practically negligible.

In the first variant the number $n_{\text {tot }} / n_{\infty}\left(t_{*}\right)$ is many times smaller than $N_{\text {tothom: }}$

$$
\frac{n_{\text {tot }}}{n_{\infty}\left(t_{*}\right)} \ll N_{\text {tothom }}
$$

Then one can simply say that the optical properties are determined according to (94) by $\widetilde{L}\left(t_{0}\right)$ and $N_{\text {tothom. Now the }}$ method of how to regulate these values is presented.

The most serious limitation of the theory is the free molecular regime of growth. It leads to the collective consumption of metastability. But when the quantity of the heterogeneous centers is small the region of influence of every heterogeneous center is large enough and it is impossible to keep the free molecular regime here. Fortunately there is a certain analogy between the situation of the collective consumption and the situation with the zones of depletion. This analogy was established in [48]. The mentioned fact was seen for the period of nucleation. Here the situation is more complex because there is no appearance of new embryos after $t_{*}$. So here there is a nucleation in the system with some very curved boundaries (the boundaries of exhausted regions) and these boundaries move rather slowly. But although the situation is another it is possible to see that the maximum of $\Psi$ can be taken in the first iteration, that is, on the base of the metastability consumption which occurs by the ideal law, that is, in approximation $\zeta=\Phi$. This approximation is good for all $\Phi$ with $d^{2} \Phi / d t^{2} \leq 0$, that is, for convex functions. But for $d^{2} \Phi / d t^{2}>0$ there appears another stabilizing factor: the rate of the supersaturation growth rapidly increases and the consumption of metastability increases. It means that the boundaries begin to move rather rapidly and the error in the position of these boundaries is not too important. So the form of the free volume can be smoothed and it is possible to come to the absence of the effects of the noncollective consumption. In any case this question forms a matter of a separate publication.

\section{List of Variables}

A: $\quad$ Surface area of the embryo

a: $\quad$ Parameter in the equation for the evolution after the heterogeneous nucleation stops

$b$ : Surplus chemical potential

$c$ : Parameter in the equation for the evolution after the heterogeneous nucleation stops

$\widetilde{c}$ : $\quad$ Parameter in the model for the free energy of the embryo on heterogeneous center

$c$ : Index to mark the critical embryo

$e: \quad$ Index to mark to equilibrium embryo

$F$ : $\quad$ Free energy of the embryo measured in the thermal units $k_{B} T$ ( $K_{B}$ is the Boltzmann constant and $T$ is the absolute temperature)

$f: \quad$ Size spectrum of the embryos

$G$ : Number of the molecules in the crystalline phase calculated in units of $n_{\infty}$

$J: \quad$ Rate of nucleation

$K: \quad$ Condensation coefficient

$K_{0}$ : $\quad$ Basis for the approximation for $K$

$k_{\pi}: \quad$ Coefficient in linearization of $\Pi$

$L_{\mid}$: Number of molecules in a new phase at the end of intensive growth

$\widetilde{L}$ : $\quad$ Mean size for the homogeneously formed embryos

$N_{\text {id het }}$ : Number of heterogeneously formed droplets calculated in approximation of the total number of the free heterogeneous centers

$n$ : $\quad$ Mean concentration of the molecules in the noncrystalline phase

$n_{1}$ : Number of sites (or the molecules) which can be the starting point for the nucleation (crystallization) formation of the embryo

$n^{e}: \quad$ Equilibrium distribution

$n_{\text {het }}$ : Number of the free heterogeneous centers (unoccupied by the supercritical embryos)

$n_{\text {near }}$ : Density of the molecules in the vicinity of the embryo

$n_{\text {tot }}: \quad$ Total number of heterogeneous centers

$n_{\infty}$ : Concentration of molecules in the noncrystalline phase at the state of the phase coexistence

$r$ : $\quad$ Linear size of the embryo

T: $\quad$ Absolute temperature

$t: \quad$ Time 
$t_{0}$ : Time of the "freezing" of the system, which is a parameter in approximation for kinetic coefficient

$t_{+}$: Moment of intensive formation of the pseudo-homogeneous embryos

$t_{*}$ : Time of appearance of embryos on the active heterogeneous centers

$t_{* *}$ : Moment of the pseudo-homogeneous formation in the presence of active heterogeneous centers

$t_{1}$ : Time of the end of the rapid growth of the embryos sizes

$v_{t}$ : Mean thermal velocity of the molecule

$W_{c}^{+}$: Kinetic factor (intensity of adsorption of the molecules by the embryo)

$Z$ : Zeldovich factor

$Z^{+}$: Truncated Zeldovich factor

$z$ : Coordinate of the spectrum of the heterogeneously formed embryos

$\Delta F$ : Height of the activation barrier of nucleation

$\Delta_{e} v$ : Width of the equilibrium zone

$\Gamma$ : $\quad$ Parameter of decompositions for the free energy, which ordinary equals the total number of the molecules inside the homogeneous critical embryo or the difference between the number of molecules in the critical embryo and the number of molecules in the equilibrium embryo

$\Gamma_{\text {het }}$ : Parameter $\Gamma$ for heterogeneous nucleation

$\mu$ : Chemical potential

$v$ : Number of molecules in the embryo

$\nu_{c}$ : Number of molecules in the critical embryo

$v_{e}: \quad$ Number of molecules in the equilibrium embryo

$\Pi$ : Activation barrier of accommodation

$\Phi: \quad$ Imaginary metastability which would be in the system when the process of the new phase formation is forbidden

$\Phi_{0}$ : Basis for linearization of $\Phi$

$\phi: \quad$ Coefficient in linearization of $\Phi$

$\Psi$ : Supersaturation that would be in a system when the heterogeneous formation is allowed and the homogeneous mechanism is forbidden

$\psi: \quad$ Parameter of linearization of $\Psi$

$\rho: \quad$ A cubic root of $\nu$

$\sigma: \quad$ Renormalized surface tension

$\tau$ : Characteristic time, parameter in the law of growth, which ordinary equals the mean time between collisions of some given molecule of the saturated disordered phase with other molecules in this phase

$\zeta$ : $\quad$ Power of metastability or the supersaturation

$\zeta_{0}: \quad$ Basis for decompositions for $\zeta$

!: Index denoting the values at the period of nucleation.

\section{Conflict of Interests}

The author declares that there is no conflict of interests regarding the publication of this paper.

\section{References}

[1] I. S. Gutzow and J. W. P. Schmelzer, The Vitreous State Thermodynamics, Structure, Rheology, and Crystallization, Springer, Heidelberg, Germany, 2nd edition, 2013.

[2] F. R. S. Lord Rayleigh, "XXXIV. On the transmission of light through an atmosphere containing small particles in suspension, and on the origin of the blue of the sky," Philosophical Magazine Series 5, vol. 47, no. 287, pp. 375-384, 1899.

[3] M. Smoluchowsky, "Molecular-kinetic theory of the opalescence of gases at the critical point," Annalen der Physik, vol. 25, pp. 205-226, 1908.

[4] A. Einstein, "Theorie der opaleszenz von homogenen flüssigkeiten und flüssigkeitsgemischen in der nähe des kritischen zustandes," Annalen der Physik, vol. 33, pp. 1275-1298, 1910.

[5] J. Cabannes, "Sur la Diffusion de la Lumière Par les Molécules des Gaz Transparents," Annales de Physique (Paris), vol. 15, pp. 5-149, 1921.

[6] J. Cabannes, La Diffusion Moleculaire de la Lumiere, Les Presses Universitaires de France, Paris, France, 1929.

[7] G. Mie, "Contributions to the optics of the turbid media, particularly of metal and colloid particles," Annalen der Physik, vol. 25, no. 3, pp. 377-445, 1908.

[8] G. Jobst, "Zur farbentheorie kolloidaler metallsuspension," Annalen der Physik, vol. 76, pp. 863-888, 1925.

[9] P. Debye, "Der lichtdruck auf kugeln von beliebigem material," Annalen der Physik, vol. 335, no. 11, pp. 57-136, 1909.

[10] J. Frenkel, Kinetic Theory of Liquids, Oxford University Press, New York, NY, USA, 1977.

[11] R. Becker and W. Doering, "Kinetische behandlung der keimbildung in übersättigten dampfen," Annalen der Physik, vol. 24, pp. 749-752, 1935.

[12] M. Volmer, "Über Keimbildung und Keimwirkung als Spezialfälle der heterogen Katalyse," Zeitschrift für Elektrochemie, vol. 35, no. 9, pp. 555-561, 1929.

[13] H. Kramers, "Brownian motion in a field of force and the diffusion model of chemical reactions," Physica, vol. 7, no. 4, pp. 284-304, 1940.

[14] J. B. Zeldovich, "On the theory of new phase formation; Cavitation," Acta Physicochimica USSR, vol. 18, pp. 1-22, 1943.

[15] F. M. Kuni, "Kintics of heterogeneous condensation. 1. Snationary state and the time of its formation," Colloid Journal, vol. 46, p. $674,1984$.

[16] A. I. Rusanov and F. M. Kuni, "Towards theory of nucleation on charged nucleus. I. Relations of general thermodynamics," Colloid Journal of the Russian Academy of Sciences, vol. 44, p. 934, 1982.

[17] A. I. Rusanov and F. M. Kuni, "Towards theory of nucleation on charged nucleus.II. Thermodynamic parameters of equilibrium embryo," Colloid journal of the Russian Academy of Sciences, vol. 44, p. 1062, 1982.

[18] A. I. Rusanov and F. M. Kuni, "Towards theory of nucleation on charged nucleus. III. Decomposition of the parameter of the droplets curvature in the field of the charged nuclei," Colloid journal of the Russian Academy of Sciences, vol. 45, p. 682, 1982. 
[19] J. W. Gibbs, The Collected Works of J.W. Gibbs, Longmans, Green, New York, NY, USA, 1928.

[20] S. Sen, M. A. Varshney, and D. Varshney, "Role of density profiles for the nonlinear propagation of intense laser beam through plasma channel," Advances in Optical Technologies, vol. 2014, Article ID 472740, 7 pages, 2014.

[21] G. O. Berim and E. Ruckenstein, "Kinetic theory of heterogeneous nucleation; effect of nonuniform density in the nuclei," Journal of Colloid and Interface Science, vol. 355, no. 1, pp. 259264, 2011.

[22] A. C. Zettlemoyer, Nucleation, Marcel Dekker, New York, NY, USA, 1969.

[23] F. F. Abraham, Homogeneous Nucleation Theory, Academic Press, New York, NY, USA, 1974.

[24] F. F. Abraham, "A reexamination of homogeneous nucleation theory: thermodynamic aspects," Journal of the Atmospheric Sciences, vol. 25, no. 1, pp. 47-53, 1998.

[25] R. C. Cammarata, "Generalized surface thermodynamics with application to nucleation," Philosophical Magazine, vol. 88, no. 6, pp. 927-948, 2008.

[26] A. Reinhardt and J. P. K. Doye, "Free energy landscapes for homogeneous nucleation of ice for a monatomic water model," The Journal of Chemical Physics, vol. 136, no. 5, Article ID 054501, 2012.

[27] F. G. Shi, "Direct measurement of free-energy barrier to nucleation of crystallites in amorphous silicon thin films," Journal of Applied Physics, vol. 76, no. 9, pp. 5149-5153, 1994.

[28] R. B. McClurg and R. C. Flagan, "Critical comparison of droplet models in homogeneous nucleation theory," Journal of Colloid and Interface Science, vol. 201, no. 2, pp. 194-199, 1998.

[29] S. Ryu and W. Cai, "Validity of classical nucleation theory for Ising models," Physical Review E-Statistical, Nonlinear, and Soft Matter Physics, vol. 81, no. 3, Article ID 030601, 2010.

[30] A. Laaksonen, V. Talanquer, and D. W. Oxtoby, "Nucleation: measurements, theory, and atmospheric applications," Annual Review of Physical Chemistry, vol. 46, no. 1, pp. 489-524, 1995.

[31] R. Evans, "The nature of the liquid-vapour interface and other topics in the statistical mechanics of non-uniform, classical fluids," Advances in Physics, vol. 28, no. 2, pp. 143-200, 1979.

[32] D. W. Oxtoby and R. Evans, "Nonclassical nucleation theory for the gas-liquid transition," The Journal of Chemical Physics, vol. 89, no. 12, pp. 7521-7530, 1988.

[33] X. C. Zeng and D. W. Oxtoby, "Gas-liquid nucleation in Lennard-Jones fluids," The Journal of Chemical Physics, vol. 94, no. 6, pp. 4472-4478, 1991.

[34] B. N. Hale, "The scaling of nucleation rates," Metallurgical transactions A: Physical metallurgy and materials science, vol. 23, no. 7, pp. 1863-1868, 1992.

[35] D. Stauffer, "Scaling theory of percolation clusters," Physics Reports, vol. 54, no. 1, pp. 1-74, 1979.

[36] K. R. Bauchspiess and D. Stauffer, "Use of percolation clusters in nucleation theory," Journal of Aerosol Science, vol. 9, no. 6, pp. 567-577, 1978.

[37] F. F. Abraham and G. M. Pound, "A reexamination of the free energy of droplet formation and dependence of surface tension on radius," Journal of Crystal Growth, vol. 6, no. 4, pp. 309-310, 1970.

[38] F. F. Abraham and G. M. Pound, "Free energy of formation of droplets from vapor and dependence of surface tension on radius," Journal of Crystal Growth, vol. 2, no. 3, pp. 165-168, 1968.
[39] W. R. Wilcox, "The relation between classical nucleation theory and the solubility of small particles," Journal of Crystal Growth, vol. 26, no. 1, pp. 153-154, 1974.

[40] W. A. Cooper and C. A. Knight, "Heterogeneous nucleation by small liquid particles," Journal of Aerosol Science, vol. 6, no. 3-4, pp. 213-221, 1975.

[41] D. Schwarz, R. Van Gastel, H. J. W. Zandvliet, and B. Poelsema, "Size fluctuations of near critical nuclei and gibbs free energy for nucleation of BDA on $\mathrm{Cu}(001)$," Physical Review Letters, vol. 109, no. 1, Article ID 016101, 2012.

[42] I. Kusaka, M. Talreja, and D. L. Tomasko, "Beyond classical theory: predicting the free energy barrier of bubble nucleation in polymer foaming," AIChE Journal, vol. 59, no. 8, pp. 30423053, 2013.

[43] H. Reiss and W. K. Kegel, "Replacement free energy and the 1/S factor in nucleation theory as a consequence of mixing entropy," Journal of Physical Chemistry, vol. 100, no. 24, pp. 10428-10432, 1996.

[44] D. Reguera and J. M. Rubi, "Nonequilibrium translationalrotational effects in nucleation," The Journal of Chemical Physics, vol. 115, no. 15, p. 7100, 2001.

[45] J. S. Langer, “Theory of nucleation rates," Physical Review Letters, vol. 21, no. 14, pp. 973-976, 1968.

[46] J. S. Langer, "Statistical theory of the decay of metastable states," Annals of Physics, vol. 54, no. 2, pp. 258-275, 1969.

[47] F. M. Kuni, A. P. Grinin, and V. B. Kurasov, "Heterogeneous nucleation in gas flow," in Mechanics of Inhomogeneous Systems, G. Gadiak, Ed., pp. 86-110, Novosibirsk, 1985.

[48] V. B. Kurasov, "Density profiles in the theory of condensation," Physica A, vol. 226, no. 1-2, pp. 117-136, 1996. 


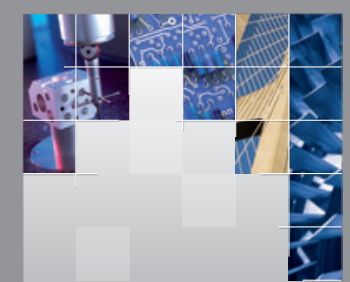

\section{Enfincering}
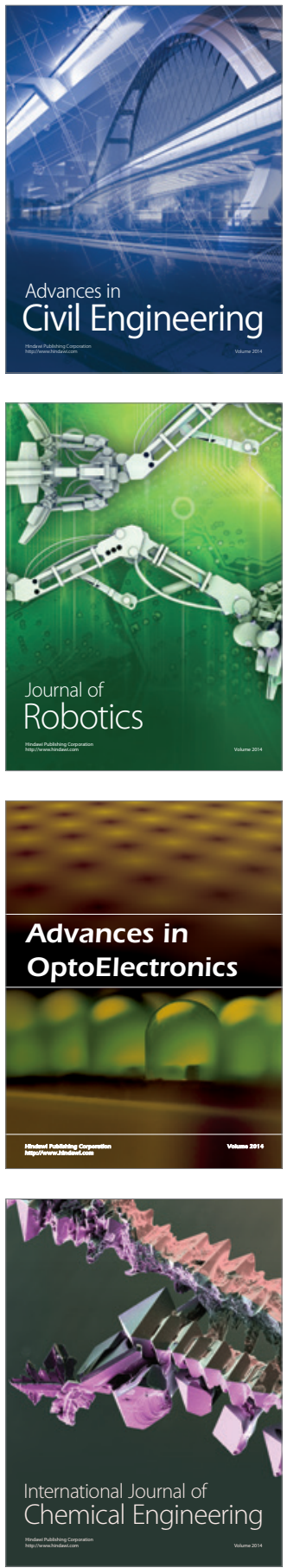

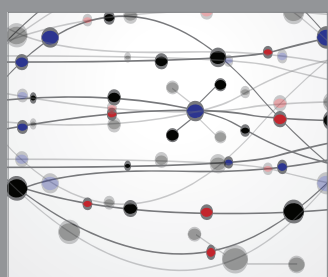

The Scientific World Journal

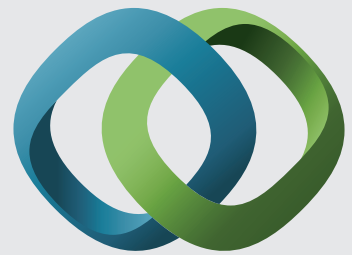

\section{Hindawi}

Submit your manuscripts at

http://www.hindawi.com
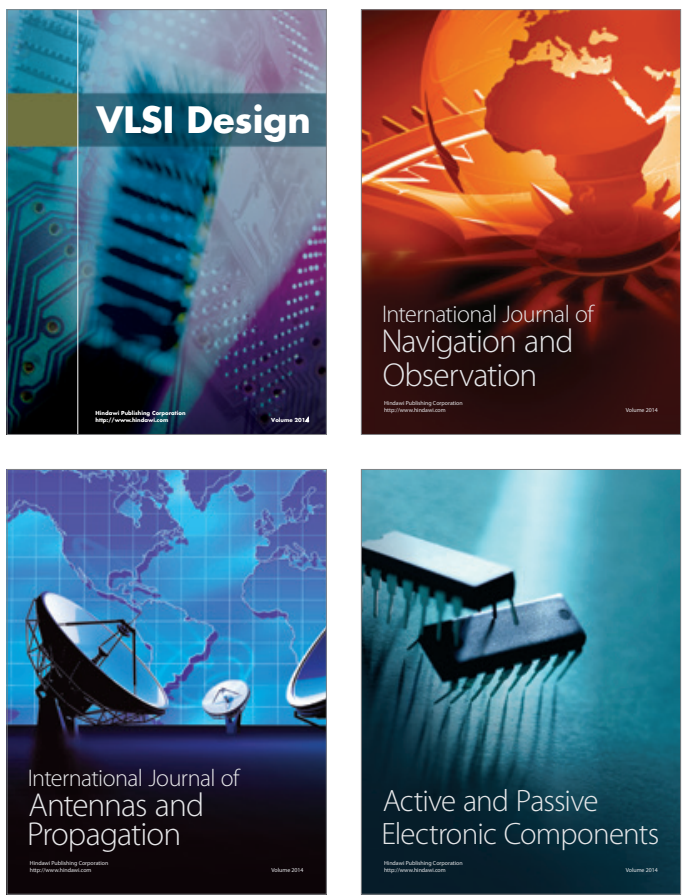
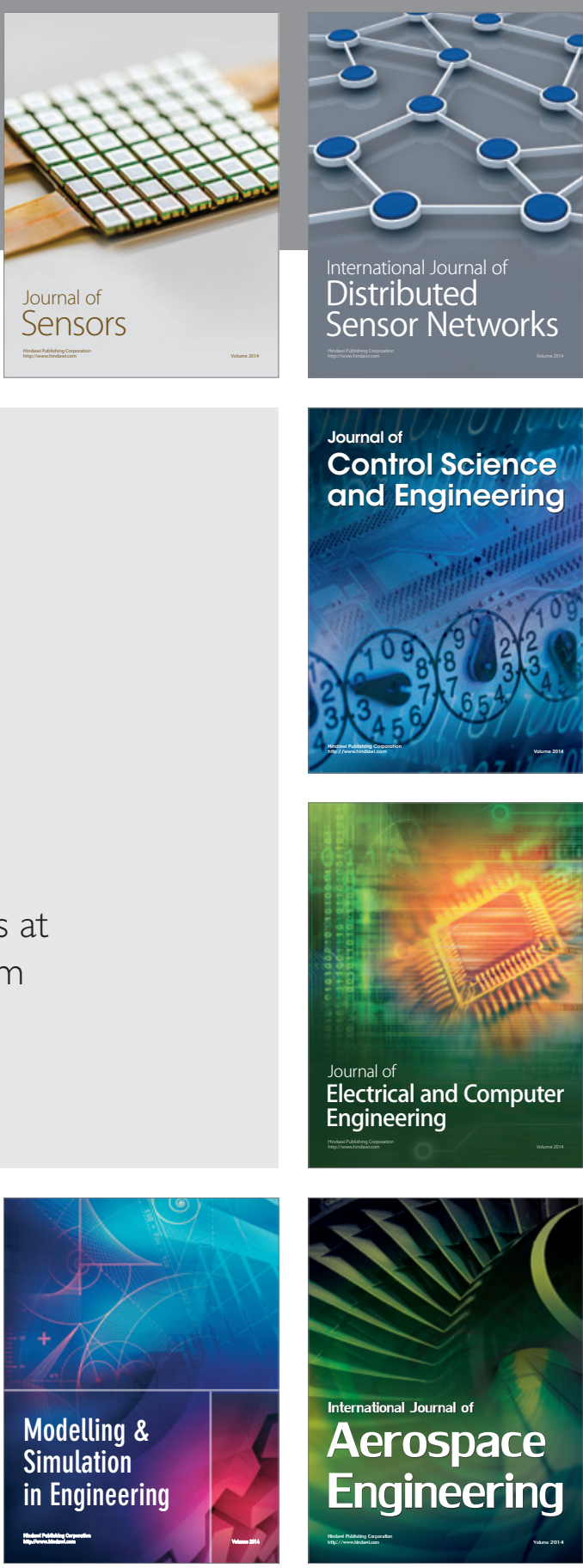

International Journal of

Distributed

Sensor Networks

Journal of

Control Science

and Engineering
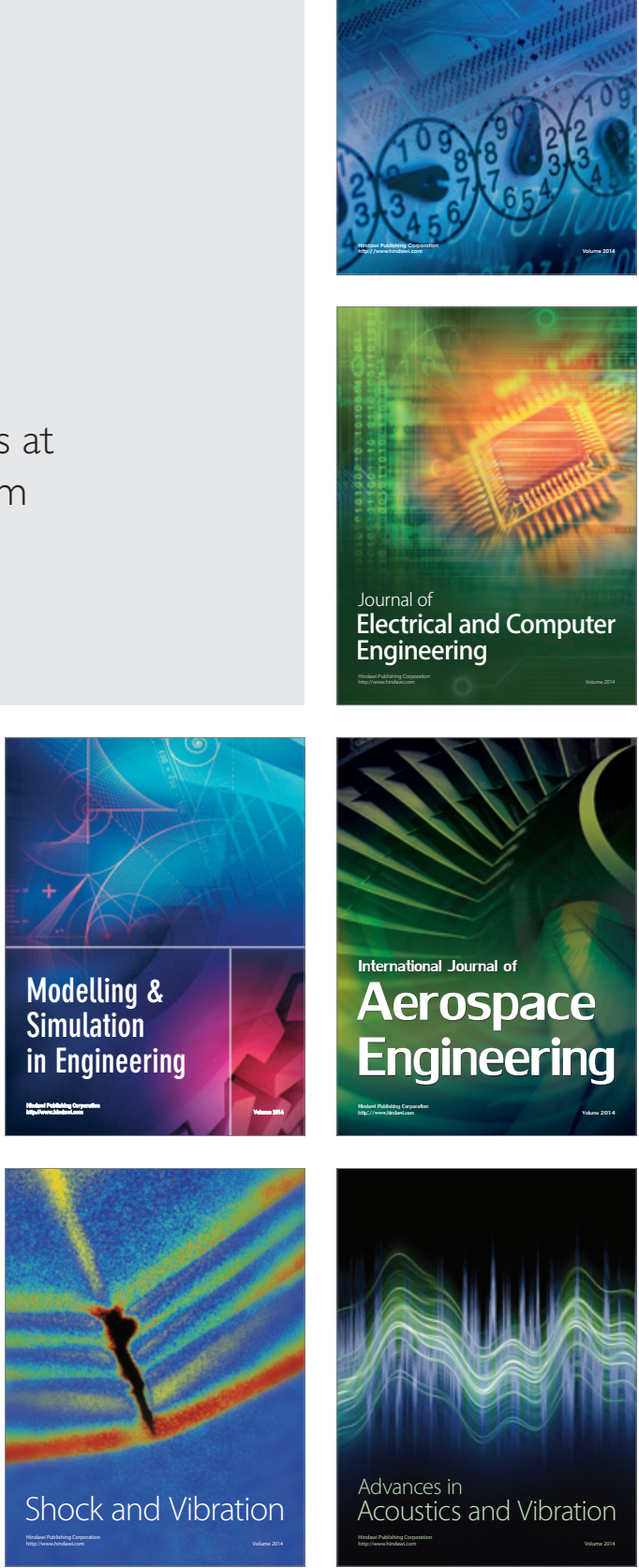